\title{
ALTENHILFE
}

\section{Schneller leben, schneller pflegen}

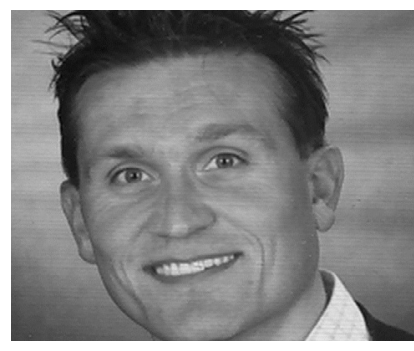

VON SASCHA M. BUCHINGER Sascha M. Buchinger ist Referent für stationäre und teilstationäre Altenhilfe beim Deutschen Roten Kreuz, Landesverband BadenWürttemberg e. V. in Stuttgart. E-Mail

sascha.buchinger@gmx.de
Die Folgen des gesellschaftlichen Wandels zeigen sich sowohl bei den Einrichtungen der Altenpflege und den Pflegekräften als auch bei den Pflegebedürftigen. In wieweit sich die gegenwärtige Situation der Pflege von der Postmoderne ableiten und sich Herausforderungen der nahen $\mathrm{Zu}$ kunft skizzieren lassen, kann an verschiedenen Parametern aufgezeigt werden.

Das postmoderne Leben, mit dem der kulturelle Wandel ab Ende der 1960er-Jahre bezeichnet wird, ist charakterisiert durch zahlreiche pluralistische, komplexe und innovative Strömungen, die ebenso vielfältige Auswirkungen auf die Gesellschaft und ihre Mitglieder haben. Ein erstes Kennzeichen der Postmoderne bildet der technische Fortschritt. In der Industrie schritt die Automatisierung des Produktionsprozesses voran, sodass die vom Menschen unabhängig gesteuerten technischen Vorgänge auf der einen Seite zur Maximierung der Fertigung, Qualitätssteigerung und physischen Entlastung der Arbeitnehmer führten. Auf der anderen Seite wurde menschliche Arbeit eingespart und es resultierte daraus ein Anstieg der Arbeitslosigkeit.

Gleichzeitig kam es zu einer weltweiten Verflechtung der Wirtschaft. Die Internationalisierung des Handels auf globalen Märkten ermöglicht es immer mehr Unternehmen, über weltweite computergestützte Logistik, Datennetze und hochtechnisierte Verkehrsmittel, auf jedem Kontinent die für sich günstigsten Produktionsstandorte zu lokalisieren und Investitionen zu tätigen. In diesem Kontext veränderte sich der sektorale Aufbau der Wirtschaft. Der primäre Bereich der Volkswirtschaft (u. a. Landwirtschaft, Fischzucht und Bergbau) wurde immer unbedeutender gegenüber dem verarbeitenden Gewerbe. Hauptsächlich kam es jedoch zu einer Tertiarisierung des Arbeitsmarktes: Der Dienstleistungssektor erfuhr einen enormen Zuwachs. Neben der Banken- oder Versicherungsbranche und der öffentlichen Verwaltung zeigt sich dieser Trend auch in der Altenhilfe.

Für die Pflege und Betreuung von hilfebedürftigen Menschen ergaben sich aus diesen Entwicklungen unterschiedliche Konsequenzen. Im tertiären Sektor spielt die Bedeutung der Muskelkraft nur noch eine untergeordnete Rolle, sodass hier vor allem eine zunehmende Bedeutung der weiblichen Erwerbstätigkeit zu verzeichnen war und ist. Obgleich die Pflege allgemein als feminisiert zu bezeichnen ist (sowohl aufseiten der Pflegekräfte als auch aufseiten der Bewohnerschaft dominiert das weibliche Geschlecht), sorgte die Verlagerung von Produktionsbereichen für freiwerdende Arbeitnehmerinnen, die in der Folge teilweise mit Unterstützung von Arbeitsagenturen oder Bildungsgutscheinen den Weg in die Altenhilfe gefunden haben. Somit stellt für viele in der Altenhilfe Tätige die pflegerische Profession nicht die Ausbildung oder den Beruf der Wahl dar (vgl. Voges 2001).

Daneben rekrutiert die Pflege schon seit langer Zeit - verstärkt nach der Vereinigung Deutschlands, der fortschreitenden Europäisierung und der Öffnung des ehemaligen Ostblocks - Mitarbeiter mit Zuwanderungsgeschichte zur Gewährleistung der Versorgungsprozesse. Fehlen auch statistische Werte, so ist dennoch die interkulturelle Öffnung, wenn auch gelegentlich unreflektiert, durch Pflegende aus der ehemaligen Sowjetunion, der Türkei und Polen oder dem einstigen Jugoslawien bereits bundesdeutscher Alltag.

Als eine Begleiterscheinung der weltweiten Krise der Wirtschaftsmärkte könnte sich mittelfristig die Attraktivität der Altenhilfe erhöhen. Angesichts massiver Kurzarbeit, Fusionen, Stellenabbau und einer zunehmenden Ausbildungsdefensive in Industrie und Handwerk könnte sich bei Jugendlichen das negative Altenhilfe-Image (u. a. DBfK 2009; ver.di 2008) zu einem krisenfesten und zukunftsträchtigen Arbeitsfeld wandeln.

\section{Demografische Trends verändern die Gesellschaft}

Zum Wachstumsmarkt Altenhilfe trägt wesentlich die demografische Entwicklung als ein weiteres Kennzeichen der Postmoderne bei. Der demografische Wandel mit dem sich signifikant verändernden Altersaufbau der Gesellschaft wird (je nach Szenario) die Anzahl der hochaltrigen Menschen über 80 Jahre ansteigen lassen (von 
derzeit $5 \%$ auf $14 \%$ bis 2060 ), für eine enorme Zunahme an Personen mit dementiellen Veränderungen sorgen und letztendlich zu einem Anstieg der Zahl an Pflegebedürftigen führen: Von 2005 bis 2020 wird eine Zunahme um nahezu 37 Prozent von 2,1 auf dann 2,9 Millionen pflegebedürftigen Menschen erwartet (Statistisches Bundesamt 2008a).

Neben den statistischen Prognosen aus gerontologischer und geriatrischer Sicht mit dem Fokus auf Pflege vollziehen sich noch weitere Veränderungen innerhalb der »Risikogesellschaft « (Beck 2003). Die Rate der Ehescheidungen bewegt sich auf einem hohen Niveau (Statistisches Bundesamt 2009a) und die neuen, von Individualisierung geprägten Lebensmodelle und Privatheitsmuster (z. B. Patchwork-Familien) führen zur De-Institutionalisierung der traditionellen Familienstrukturen. Feminismus und die Loslösung aus vorhandenen Bedingungen und Traditionen ermöglichen dem Einzelnen eine umfangreiche Wahlfreiheit der Lebensgestaltung.

Um die Chancen der Pluralität nutzen zu können, muss ein Individuum über Bildung, Arbeitsmarktpartizipation, Mobilität, Flexibilität und Konsumbereitschaft verfügen. Der Preis für einen hohen Lebensstandard spiegelt sich dann u. a. im kontinuierlichen Geburtentief (Frejka 2008; Statistisches Bundesamt 2009a), in der zunehmenden Anzahl der Single-Haushalte (Statistisches Bundesamt 2009a) und in der Tendenz zu multilokalen Mehrgenerationenfamilien wider, in denen die Mitglieder der verschiedenen Generationen verstreut an weit entfernten Orten leben (Peuckert 2004).

Wenn die eigenen Interessen die familiären Pflegeanforderungen überlagern, wird letztendlich die tradierte Reziprozitätsnorm zwischen den Generationen infrage gestellt: Je höher Status oder Bildungsabschluss und je moderner der Lebensentwurf oder die Interpretation der Frauenrolle (u. a. berufliche Selbständigkeit), desto ausgeprägter ist die Präferenz einer professionellen Unterstützung durch Pflegeheime (Blinkert/Klie 2008). Somit bleibt zu konstatieren, dass sich das familiale Pflegepotenzial und die familiären Unterstützungsbeziehungen reduziert haben und sich dieser Prozess fortsetzen wird.

Darüber hinaus resultiert sowohl aus sozioökonomischen wie auch biologischen Faktoren eine kontinuierlich ansteigende Lebenserwartung, die zu einer extremen Form der intergenerativen Pflege führt. Plakativ formuliert: 100-jährige Pflegebedürftige werden von 75-jährigen Kindern und Angehörigen gepflegt. Dass deren Leistungsfähigkeit und Pflegebereitschaft im Zusammenhang mit der pflegerischen Kosten-Nutzen-Bilanz nur begrenzt abgerufen werden kann, erscheint plausibel.
Auch im Kontext professioneller Unterstützung kommt es durch die demografische Entwicklung zu folgeträchtigen Veränderungen. Zur rechnerischen Konstanterhaltung der derzeitigen Bevölkerung ist eine Geburtenrate von 2,1 notwendig. Erreicht wird jedoch schon seit längerer Zeit nur eine Fertilitätsquote von 1,37 (Statistisches Bundesamt 2009b), die Gesamtbevölkerung in Deutschland und weiten Teilen Europas wird also schrumpfen. Parallel wird sich auch die Zahl der weiblichen Erwerbspersonen (zwischen 35 und 55 Jahren) von derzeit 10,4 Millionen auf rund 5,9 Millionen im Jahre 2050 nahezu halbieren (Hackmann 2009). Für den zukünftigen Wachstumsmarkt Altenhilfe wird aber ein ren tarifliche Steigerungen von bis zu elf Prozent zu verzeichnen, was in möglichen Pflegesatzverhandlungen eine entsprechende Berücksichtigung findet. Um die gesteigerten Vergütungsausgaben zu refinanzieren, wird der Pflegesatz einer Einrichtung entsprechend erhöht und die Bewohner oder der Sozialhilfeträger haben einen verteuerten Eigenbeitrag zu schultern.

Neben den Personalkosten haben ordnungsrechtliche Vorgaben das Potenzial, die Heimentgelte zu verteuern. Beispielsweise wurde in Baden-Württemberg den Pflegebedürftigen der Anspruch auf ein Einzelzimmer gesetzlich eingeräumt, sodass zukünftig nach Verordnung ausschließlich diese Zimmer neu gebaut werden dürfen

\section{"Neue Unterstützungsformen sind notwendig, wenn 100-jährige Eltern von ihren 75-jährigen Kindern gepflegt werden sollen"}

immenser Personalbedarf konstatiert, der teilweise schon heute nicht mehr gedeckt werden kann. Aktuell können regional spezifisch vakante Fachkraftstellen nicht besetzt werden, sodass vielerorts schon von einem Fachkräftemangel und Pflegenotstand gesprochen wird. Auch die Zahl der Auszubildenden ist in den pflegerischen Berufen seit Jahren rückläufig.

Sollten sich diese Prozesse verschärfen, werden neben personal- und bildungspolitischen auch konzeptionelle und vor allem leistungs- und ordnungsrechtliche Überlegungen notwendig, um die Versorgung weiterhin aufrecht erhalten zu können.

Als eine weitere Konsequenz resultiert aus fehlendem Fachpersonal möglicherweise eine Verteuerung der Pflege durch erhöhte Personalkosten. Bei der Wohlfahrtspflege werden derzeit rund 77 Prozent der stationär Beschäftigten von ihren Trägern außertariflich vergütet (verdi-drei 2008). Hinzu addieren sich 4.300 Pflegeheime, deren Pflegeakteure unter privater Trägerschaft oftmals ebenso untertariflich entlohnt werden. Die quantitative Verringerung des notwendigen pflegerischen »Humankapitals « sowie die Verknappung qualifizierter Kräfte übt angesichts des schon eingetretenen Verdrängungswettbewerbes auf die Pflegeheimträger Druck aus, Mitarbeiter abzuwerben und materielle Anreize zu schaffen.

Personalkosten bilden mit rund 80 Prozent den größten Kostenfaktor im Etat einer Einrichtung. In den letzten zwei Jahren wa- oder Doppelzimmer umgestaltet werden müssen. Letztendlich kommt es auch hier durch die Einrichtungen zu einer Anpassung der Investitionskosten nach oben (COCON CONCEPT 2009).

\section{Wissen durch Medien}

Massenmedien für die Verbreitung von Nachrichten, Wissen, Unterhaltung und Kultur sind ein weiteres Kriterium der postmodernen Entwicklung. Die Medialisierung mit einem Datenfluss in virtuellen Welten durch unbegrenzten Informationszugang führt vermehrt zu einer Entfernung von der Wirklichkeit und zu einer Zunahme der Informationen aus zweiter Hand. Durch Printmedien, Telefon, Fax, Fernsehen und vor allem Internet ist der Mensch in der Lage, Erfahrungen, Zeitvertreib, Einkauf, Bildung und Kommunikation zu bewerkstelligen, ohne das eigene Heim verlassen oder mit anderen Menschen persönlich in Kontakt treten zu müssen.

Gleichzeitig hat das produzierte Wissen eine immer kürzere Halbwertszeit, wird immer komplexer und spezialisierter. Für den Professionalisierungsprozess der Altenhilfe bedeutet dies die Entwicklung eines eigenen Bewusstseins mit einer fortschreitenden Akademisierung und Verwissenschaftlichung und der Notwendigkeit für die pflegerischen Akteure zur permanenten Fortund Weiterbildung.

Die unaufhaltsame Entwicklung von Maschinen und automatisierten Gegen- 
Literatur:

Avivre Consult (2007): Pflegemarkt 06, Bad Homburg.

Beck, Ulrich (2003): Risikogesellschaft. Auf dem Weg in eine andere Moderne, Frankfurt am Main.

BGW Berufsgenossenschaft für Gesundheitsdienst und Wohlfahrtspflege (2007): Altenpflege in Gefahr: Viele Pfleger ständig krank, Pressemitteilung 6.2.2007, Hamburg. Blinkert, Baldo/Klie, Thomas (2004): Gesellschaftlicher Wandel und demografische Veränderungen als Herausforderung für die Sicherstellung der Versorgung von pflegebedürftigen Menschen. In: Sozialer Fortschritt 53, S. 319-325. Blinkert, Baldo/Klie, Thomas (2008): Soziale Ungleichheit und Pflege, APuZ 12-13/2008.

BMFSFJ Bundesministerium für Familie, Senioren, Frauen und Jugend (2008): Möglichkeiten und Grenzen selbständiger Lebensführung in stationären Einrichtungen (MuG IV), Berlin. BMG Bundesministerium für Gesundheit

(2009): Gesetzliche Krankenversicherung. Krankenstand 1979 bis 2008 und Januar bis Oktober 2008, Berlin.

Buchinger, Sascha M. (2009): Kommt der Pflegenotstand? In: Heim + Pflege, Nr. 11, 40. Jg., S. 296-301.

COCON CONCEPT (2009): Von 30 auf 100. 100\% Einzelzimmer als Mindeststandard für stationäre Pflegeeinrichtungen in Baden-Württemberg, Berlin.

DBfK Dt. Berufsverband für Pflegeberufe (2009): Wie sieht der Pflegealltag wirklich aus? Fakten zum Pflegekollaps. Ausgewählte Ergebnisse der DBfK-Meinungsumfrage 2008/o9, Berlin.

Deutsche Bank Research (2009): Demografie spezial. Deutsche Pflegeversicherung vor massiven Herausforderungen, Aktuelle Themen 442, Frankfurt am Main.

Dörner, Klaus (2003): Der gute Arzt. Lehrbuch der ärztlichen Grundhaltung, Stuttgart.

Frejka, Tomas (2008): Weite Teile Europas dauerhaft im Geburtentief. In: Max-Planck-Institut für demografische Forschung: Demografische Forschung, Nr. 4 / 2008, 5. Jg., Rostock.

Görgen, Thomas (1999): Belastungen, Konflikte und Gewalt in stationären Altenhilfeeinrich-

tungen. Ergebnisse einer Befragung von Pflegekräften, Gießen.

Hackmann, Tobias (2008): Pflege als neuer Beschäftigungsmotor? Entwicklung des Personalbedarfs in ambulanter und stationärer Pflege, Internet http://www.vwl.uni-freiburg.de/fakultaet/fiwil/downloads/ aktuelles/pflege.pdf. Hackmann, Tobias (2009): Simulationsmodell zur langfristigen Entwicklung des Pflegebedarfs in Deutschland, Vortrag Dt. Pflegekongress 29.5.2009, Berlin.

Hamburger Abendblatt (2009): Immer weniger Fachpersonal in Hamburg, 18.1.2009, Internet http://www.abendblatt.de/hamburg/article593094/Immer-weniger-Fachpersonal-inHamburg.html.
Hasselhorn, Hans-Martin/Müller, Bernd Hans/Tackenberg, Peter (2005): Berufsausstieg bei Pflegepersonal, Bremerhaven.

Hirsch, Rolf D. (1993): Balintgruppe und Supervision in der Altenarbeit, München.

Hofmann, Herbert (2006): Altenpflege und Soziale Dienste - wachsender Bedarf bei fehlenden Mitteln. In: ifo Schnelldienst 15 /2006, 59. Jg., München.

Institut für Demoskopie Allensbach (2009): Pflege in Deutschland, Hamburg.

IW Institut der deutschen Wirtschaft (200):

Pressemitteilung Nr. 55, 29.12.2009, Köln.

IWAK Institut für Wirtschaft, Arbeit und Kultur (2009): Berufsverläufe von Altenpflegerinnen und Altenpflegern, Frankfurt am Main.

Landtag von Baden-Württemberg (1997): Pflegeversicherung und stationäre Altenhilfe, Drucksache 12/2080, Stuttgart.

Laslett, Peter (1995): Das Dritte Alter, Weinheim.

Luy, Marc (2006): Perspektiven für die zukünftige Entwicklung der Lebenserwartung, Rostocker Zentrum - Diskussionspapier, No.4, Rostock.

Marseille-Kliniken AG (2009): Geschäftsbericht 2008, Hamburg, Internet http://www.marseille-kliniken.de/global/downloads/pdf/mkag qualitaetsberichtog.pdf.

Mollenkopf, Heidrun (2009): Assistenzsysteme - Voraussetzungen und Handlungsbedarf, Vortrag DKE Tagung, Internet http://www.dke.de/ NR/rdonlyres/4619EC2E-268A-4A2O-B7Co257DB4246F39/28961 /Mollenkopf1.pdf. Oeppen J./Vaupel; James W. (2002): Broken limits to life expectancy. In: Science, 296, Nr. 5570, S. 1029-1031.

Peuckert, Rüdiger (2004): Familienformen im sozialen Wandel, Wiesbaden.

Robert-Koch-Institut (2005): Übergewicht und Adipositas. Gesundheitsberichterstattung des Bundes, Berlin.

Robert-Koch-Institut (2007): Altersdemenz. Gesundheitsberichterstattung des Bundes, Heft 28, Berlin.

Robert-Koch-Institut (2007): Epidemiologisches Bulletin: Übergewicht und Adipositas in Deutschland, Nr. 18, 4. Mai 2007, Berlin.

Statistisches Bundesamt (2008a): Demografischer Wandel in Deutschland, Heft 2, Auswirkungen auf Krankenhausbehandlungen und Pflegebedürftige im Bund und in den Ländern, Wiesbaden.

Statistisches Bundesamt (2008b): Bildung und Kultur, Fachserie 11, Reihe 2, Wiesbaden.

Statistisches Bundesamt (2009a): Statistisches Jahrbuch 2009, Wiesbaden, S. 46.

Statistisches Bundesamt (2009b): Geburtenentwicklung, Wiesbaden.

Statistisches Bundesamt (2009c): Pflegestatistik 2007, Wiesbaden.

Statistisches Bundesamt (2009d): Bevölkerung Deutschlands bis 2060, Wiesbaden.

Universität Halle-Wittenberg (2009): For- schungsstudie zur Verweildauer von Pflegekräften in Rheinland-Pfalz, Berichte aus der Pflege Nr. 12, Mainz.

Vaupel, J. w./Christensen, K./Фrstavik, K. H. (2001): The $X$ chromosome and the female survival advantage. In: Weinstein, M./Hermalin, A. I./Stoto, M. A. (Eds.): Population health and aging, New York. Ver.di (2008): Arbeitsqualität aus Sicht von Altenpfleger/innen, Berlin.

Ver.di (2009a): Fachkräftemangel in der Altenpflege, Infopost 84/2009, Internet http://potsdam-nwb.verdi.de/fachbereiche/03_gesundheit_soziale_dienste_wohlfahrt_und_kirchen/f achkommission_pflege/data/infopost_84-09fachkraeftemangel.pdf.

Verdi-drei (2008): Arme Brüder, Informationen für den Fachbereich Gesundheit, Soziale Dienste, Wohlfahrt und Kirchen, Nr. 28 Dezember 2008, Berlin

Voges, Wolfgang (2001): Pflege alter Menschen als Beruf. Soziologie eines Tätigkeitsfeldes, Opladen/Wiesbaden.

Zawatka-Gerlach, U. (2009): Altersarmut steigt dramatisch. In: Tagesspiegel, Nr. 68 23.03.2009, Internet http://www.tagesspiegel.de/berlin/Altersarmut;art270,2757550.

Ziegler, Uta / Doblhammer, Gabriele (2008): Bereits 1,2 Millionen Demenzerkrankte in Deutschland. In: Max-Planck-Institut für demografische Forschung: Demografische Forschung, Nr. 4 / 2008, 5. Jg., Rostock. Zimber, Andreas/Weyerer, Siegfried (1998): Stress in der stationären Altenpflege. Arbeitsbedingungen und Arbeitsbelastungen in $\mathrm{Hei}-$ men - eine Verlaufsstudie, Köln. 
ständen führt zusätzlich zu deren alltäglichen Gebrauch und einer Integration in das gegenwärtige Leben. Der gewohnte Zugang zu jeglicher Art von Technik erhöht bei den Älteren von morgen die Akzeptanz technisierter Unterstützungsmöglichkeiten und die Bereitschaft zur Nutzung (i. S. von Technik-Compliance) derartiger Systeme im Alter. In diesem Kontext schreitet die Einführung intelligenter Assistenzsysteme zur technischen Kompensation von Beeinträchtigungen und Kompetenzdefiziten voran (»Ambient Assisted Living «), wobei derzeit die Feminisierung des Alters (mit einer geringen Technikerfahrung) und noch zahlreiche Technikbarrieren die Handhabung v. a. im häuslichen Kontext erschweren (Mollenkopf 2009). Doch auch in der stationären Versorgung nimmt die Bedeutung moderner Lifter, computergestützter Dokumentationssysteme, Sensormatratzen, Funkfingern u.v.a.m. zu.

Eine weitere Folge der postmodernen Technisierung, Lebensgestaltung und Informationsflut zeigt sich im Zerfall und Verfall von familiären, nachbarschaftlichen und informellen Milieus. Im privaten Nahbereich nimmt die Bedeutung von Tradition und Religion immer weiter ab. Der Verlust an traditionellen Verbindungen sowie die Pluralisierung und Multikulturalisierung der Gesellschaft dünnt das Interesse an Vereinen, Parteien, Landfrauen, Genossenschaften oder Kirchen mit all den impliziten Verpflichtungen und Aufgaben aus.

Daneben konzentriert sich das eigene Engagement mehr auf sich selbst als auf den nachbarschaftlichen Nahbereich. Überzeugungen wie Gemeinschaftsgefühl, Altruismus, Nächstenliebe sowie das solidarische Handeln und Denken gehen verloren. Auch hier resultiert letztendlich der Trend, die Versorgung des »Vierten Alters (Laslett 1995) im öffentlichen Sozialraum zu institutionalisieren. Generell dürfte die rechtlich vorgegebene Devise "ambulant vor stationär « nicht mehr aufrecht zu erhalten sein - vielmehr ist davon auszugehen, dass sich ein Heim-Sogeffekt oder ein »Trend zur Verheimung « einstellt.

Das Auseinanderdriften von Arm und Reich ist eine nächste Entwicklung des postmodernen Zeitalters. Als Anzeichen können die rückläufige Lohnquote und die enorm angestiegene Ertragsquote aus Einkommen und Vermögen gewertet werden. Hier deutet sich die unaufhörliche finanzielle Schieflage der Sozialversicherungssysteme an, die sich alle aus den Lohnabgaben speisen. Als weitere Indikatoren sind befristete Arbeitsverträge, Teilzeitbeschäftigung, außertarifliche Bezahlung oder Minijobs zu sehen.

Für die Altenhilfe ergeben sich sowohl für die Leistungsanbieter (Pflegeeinrichtungen) als auch für die Leistungsträger (Pflegekassen, Kommunen als Sozialhilfeträger) entsprechende Folgen. Da die Personalquote aus betriebswirtschaftlichen Überlegungen an die Pflegeplatzbelegung geknüpft ist, sind die Träger im Zuge schwankender Auslastungszahlen an eine möglichst flexible Personaleinsatzplanung gebunden. Diese lässt sich überwiegend mit Teilzeitarbeitskräften gewährleisten, sodass in der stationären Altenhilfe trotz dem personellen Zuwachs immer weniger Vollzeitstellen zu besetzen sind.

Daneben gewinnt der Faktor Altersarmut wieder an Bedeutung. Das gesunkene Lohnniveau, die zurückliegenden Rentenreformen oder Phasen der Langzeitarbeitslosigkeit erhöhen die Zahl der pflegebedürftigen Senioren, die zur Deckung der Pflegeheimkosten auf Leistungen der Sozialhilfe angewiesen sind. Mittlerweile sind schon manche Kommunen per Kreistagsbeschluss dazu übergegangen, eine sogenannte Belegungssteuerung vorzunehmen (so beispielsweise die Landkreise Main-Tauber-Kreis und Schwäbisch-Hall in BadenWürttemberg). Über Gebietsaufteilung oder Kostenranking wird den (auch in naher Zukunft absehbaren) Inanspruchnehmern von Leistungen der Sozialhilfe und Kriegsopferfürsorge die Wahl des Heimplatzes (zumutbar) eingeschränkt, indem eine finanzielle Unterstützung bei den Heimkosten nur in kostengünstigen Pflegeheimen (im Doppelzimmer) erfolgt: Das Leben in Einrichtungen mit unverhältnismäßigen Mehrkosten (z. B. durch Einzelzimmer) wird nur in begründeten Ausnahmefällen ermöglicht.
Die allgemeine Beschleunigung des Lebens ist ebenfalls eine postmoderne Errungenschaft. Die geografische Mobilität zeigt sich heute privat aber vor allem beruflich ungebremst: Züge sind meist voll, auf den Autobahnen herrscht teilweise Dauerstau und das Flugzeug als innerkontinentales Fortbewegungsmittel ist längst Bestand des täglichen Lebens. Während die arbeitsbedingten Mobilitätsanforderungen sich über zeitintensive Auslandsaufenthalte, tägliche und wöchentliche Pendelstrecken oder häufige Geschäftsreisen erstrecken, werden in der Freizeit Wochenend-Trips nach Mallorca, republikweite Verwandtschaftsbesuche oder ausufernde touristische Tagesausflüge unternommen.

Bei vielen mündet diese Mobilität in $\mathrm{Ru}$ helosigkeit, Stress oder Krankheit und der Sehnsucht nach Entschleunigung bei einer eingeschränkten Erholungsfähigkeit. Denn gleichzeitig plagen sich immer mehr Arbeitnehmer mit einer Arbeitsplatzunsicherheit oder der Angst vor einem Arbeitsplatzverlust und sorgen für das Phänomen des »Präsentismus «: Trotz Krankheit wird der täglichen Berufstätigkeit und Arbeit nachgegangen. So sanken die Krankenstände seit 1970 von 5,6 auf 3,37 Prozent im Jahr 2008 (BMG 2009), was sich nicht nur auf veränderte Arbeits- und Beschäftigungsbedingungen oder die Fortschritte in Medizin und Gesundheitsvorsorge begründen lässt.

Dementsprechend werden die Anforderungen und Belastungen in Pflegeheimen zunehmen, denn diese Stressoren addieren sich zu den schon vorhandenen ungünstigen Arbeitsbedingungen wie Schichtdienst und Wochenendarbeit, ständiger Zeitdruck, fehlende Anerkennung, permanente Überforderung (fachlich, zeitlich und emotional), geringe soziale Unterstützung und Aufstiegschancen, wenig Selbstbestimmung und unzureichende Qualifikation für die zu verrichtende Arbeit.

Zusätzlich verändert sich die zu versorgende Klientel. Das Heimeinzugsalter liegt bei weit über 80 Jahren, viele der Pflegebedürftigen wollen gar nicht in ein Pflegeheim, die Mehrheit der Bewohnerschaft weist eine erheblich eingeschränkte Alltags-

\section{Neun Parameter: Was die stationäre Altenhilfe erwartet}

(1) multiethnische Teamzusammensetzungen

(2) Alten pflegeprofession als zweite Wahl

(3) Anstieg der Nachfrage nach stationärer Versorgung - demografische Entwicklung

- Singularisierung und Pluralisierung

- Reduzierung des familialen Pflegepotenzials

(4) perspektivischer Fachkraftmangel und Pflegenotstand
(5) Verteuerung der Pflegesatzengelte und der Heimunterbringungskosten

(6) Belegungssteuerung durch Leistungsträger

(7) pflegerische Technisierung (v. a. im häuslichen Kontext)

(8) komplexere Arbeitsbedingungen

(9) rückläufige Professionalisierung

Sascha M. Buchinger 
kompetenz auf, die behandlungspflegerischen Anforderungen und das Körpergewicht steigen und die Verweildauer sinkt zunehmend: Immer mehr Pflegebedürftige müssen rekrutiert, administrativ erfasst und für kurze Zeit professionell betreut werden. Hinzu addieren sich umfangreiche externe Prüfintervalle durch Heimaufsicht, Medizinischen Dienst der Krankenkassen, Zertifizierungsunternehmen u. v. a. m. Schon jetzt liegt der krankheitsbedingte Ausfall bei Pflegekräften in der Altenhilfe über dem Durchschnitt (BGW 2007).

Angesichts der Fülle an immer komplexer werdenden Rahmenbedingungen könnte sich der personelle Wechsel bei den Pflegeakteuren intensivieren. Die Mehrheit der Erhebungen gehen bei der Verweildauer von einer relativ hohen Fluktuation aus. Dementsprechend sind schon unterschiedliche Aktionen angelaufen, um zum einen die Attraktivität des Berufsbildes für neue Kräfte und Auszubildende zu erhöhen. Zum anderen sollen die Rahmenbedingun- gen verbessert werden, um vor allem den Verbleib von älteren Pflegeakteuren in der Altenhilfe zu sichern. Unter Umständen kommt es durch die immer differenzierteren Angebote und Tätigkeitsfelder in der Altenhilfe und die fortschreitende Akademisierung auch zu einem Ende der Professionalisierung im stationären Bereich: Die qualifizierten Kräfte ziehen sich mittelfristig aus dem operativen Bereich zurück.

\section{Fazit}

Die Erschwernisse für die familialen Pflegepotenziale und die daraus resultierende Abnahme der Hilfen im häuslichen Umfeld bedeuten für die stationäre Versorgung eine äußerst optimistische Prognose. Die Chancen für den institutionellen Wachstumsmarkt sind aber auch mit erheblichen Risiken verbunden. Denn der sich bereits andeutende Mangel an Fachpersonal und Nachwuchskräften bei einer rückläufigen Entwicklung der weiblichen Erwerbspersonen und sich im- mer weiter erschwerenden Arbeitsbedingungen bei einer ungenügenden Entlohnung (Stichwort Mindestlohndebatte) könnten dafür sorgen, dass die immense Nachfrage nicht adäquat befriedigt werden kann.

Multiethnische Teamkonstellationen und umfangreiche personalpolitische Initiativen sind vielschichtige Herausforderungen für die Pflegeheimträger, die in letzter Konsequenz die Entgelte verteuern werden. Da alle auf diesem Markt tätigen Akteure vor diesem Problem stehen und der Wandel der Gesellschaft nicht aufzuhalten sein wird, relativiert sich jedoch zumindest diese Sorge.

Alle Initiativen, Innovationen oder Konzeptionen zur Optimierung der Altenhilfeversorgung sind zu begrüßen. Eine nachhaltige und zukunftsträchtige Entwicklung im stationären Bereich sollte sich angesichts der Errungenschaften der Postmoderne aber zunehmend auf die dort Tätigen fokussieren. Ansonsten lässt sich dieses heutige System nicht aufrecht erhalten.

\section{Dritter Sektor im Wandel}

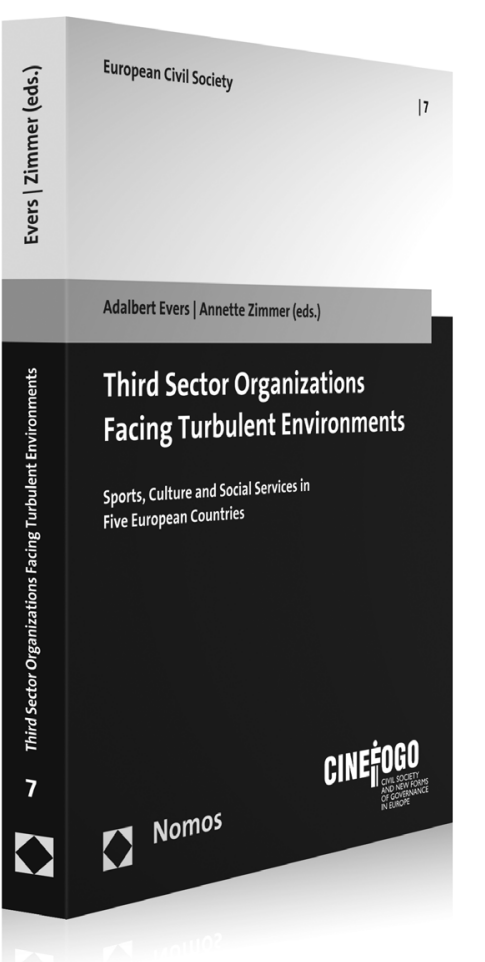

\section{Third Sector Organizations Facing Turbulent Environments}

Sports, Culture and Social Services in Five European Countries

Herausgegeben von Prof. Dr. Adalbert Evers

und Prof. Dr. Annette Zimmer

2010, 337 S., brosch., 49,- - , ISBN 978-3-8329-5421-5

(European Civil Society, Bd. 7)

In fünf europäischen Ländern wurde für die Bereiche Sport, Kultur und Sozialarbeit untersucht, wie Organisationen aus dem Dritten Sektor mit veränderten Bedingungen umgehen. Ein starker Trend hin zu Polarisierung konnte festgestellt werden: kleine, machtlose Graswurzelbewegungen auf der einen Seite und mächtige unternehmerisch geführte Organisationen auf der anderen Seite. 